CUBO A Mathematical Journal

Vol.19, No 03, (15-29). October 2017

\title{
Periodicity and stability in neutral nonlinear differential equations by Krasnoselskii's fixed point theorem
}

\author{
Bouzid Mansouri ${ }^{1}$, Abdelouaheb Ardjouni ${ }^{1,2}$ and Ahcene Djoudi ${ }^{3}$ \\ ${ }^{1}$ Faculty of Sciences, Department of Mathematics \\ Univ Annaba, P.O. Box 12, Annaba 23000, Algeria \\ ${ }^{2}$ Faculty of Sciences and Technology, Department of Mathematics and Informatics \\ Univ Souk Ahras, P.O. Box 1553, Souk Ahras, 41000, Algeria \\ ${ }^{3}$ Applied Mathematics Lab, Faculty of Sciences, Department of Mathematics \\ Univ Annaba, P.O. Box 12, Annaba 23000, Algeria \\ mansouri.math@yahoo.fr, abd_ardjouni@yahoo.fr
}

\section{ABSTRACT}

The nonlinear neutral functional differential equation with variable delay

$$
\begin{aligned}
& \frac{d}{d t} u(t)-q(t) \frac{d}{d t} g(u(t-r(t))) \\
& =p(t)-a(t) u(t)-a(t) q(t) g(u(t-r(t)))-b(t) f(u(t))+b(t) q(t) f(u(t-r(t))) .
\end{aligned}
$$

is investigated. By using Krasnoselskii's fixed point theorem we obtain the existence and the asymptotic stability of periodic solutions. Sufficient conditions are established for the existence and the stability of the above equation. Our results extend some results obtained in the work [19].

\section{RESUMEN}

La ecuación diferencial funcional no-lineal neutral con retardo variable

$$
\begin{aligned}
& \frac{d}{d t} u(t)-q(t) \frac{d}{d t} g(u(t-r(t))) \\
& =p(t)-a(t) u(t)-a(t) q(t) g(u(t-r(t)))-b(t) f(u(t))+b(t) q(t) f(u(t-r(t))) .
\end{aligned}
$$

es investigada. Usando el teorema del punto fijo de Krasnoselskii obtenemos la existencia y la estabilidad asintótica de las soluciones periódicas. Se establecen condiciones suficientes para la existencia y la estabilidad de soluciones de la ecuación anterior. Nuestros resultados extienden algunos de los resultados obtenidos en [19].

Keywords and Phrases: Fixed point, periodic solutions, stability, neutral differential equations

2010 AMS Mathematics Subject Classification: Primary 34K13, 34A34; Secondary 34K30, $34 \mathrm{~L} 30$ 


\section{Introduction}

Delay differential equations have received increasing attention during recent years since these equations have been proved to be valuable tools in the modeling of many phenomena in various fields of

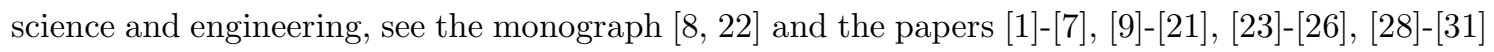
and the references therein.

Ding and Li [19] discussed the existence and the stability of periodic solutions for the following neutral functional differential equation

$$
\begin{aligned}
& \frac{d}{d t} u(t)-q \frac{d}{d t} u(t-r) \\
& =p(t)-a u(t)-a q u(t-r)-b f(u(t))+b q f(u(t-r)) .
\end{aligned}
$$

By employing Krasnoselskii's fixed point theorem, the authors obtained existence and asymptotic stability results for periodic solutions.

In this paper, we are interested on the existence and the asymptotic stability of periodic solutions of the following nonlinear neutral differential equation

$$
\begin{aligned}
& \frac{d}{d t} u(t)-q(t) \frac{d}{d t} g(u(t-r(t))) \\
& =p(t)-a(t) u(t)-a(t) q(t) g(u(t-r(t)))-b(t) f(u(t))+b(t) q(t) f(u(t-r(t))),
\end{aligned}
$$

where $r$ is positive differentiable function, and $q, p, a, b, f$ and $g$ are continuously differentiable functions. To show the existence and the asymptotic stability of periodic solutions, we transform (1.2) into integral equation and then use Krasnoselskii's fixed point theorem. The obtained integral equation split in the sum of two mappings, one is a contraction and the other is compact. It is easy to see that (1.2) reduce to (1.1) when, $q(t)=q, a(t)=a, b(t)=b, r(t)=r$ are constants and $g(u(t-r(t)))=u(t-r)$ with $|q|<1$. Then, the results obtained here extend some results of the work of Ding and Li [19].

\section{Existence of periodic solutions}

In this section, $C^{1}(\mathbb{R})$ or $C(\mathbb{R})$ denotes the set of all continuously differentiable functions or all continuous functions $\phi: \mathbb{R} \rightarrow \mathbb{R}$ respectively. $C_{\omega}=\{\phi \in C(\mathbb{R}), \phi(t+\omega)=\phi(t)\}$ is with the supremum norm $\|\cdot\|_{0}$ and $C_{\omega}^{1}=C^{1}(\mathbb{R}) \cap C_{\omega}$ is with the norm $\|\phi\|_{1}=\|\phi\|_{0}+\left\|\phi^{\prime}\right\|_{0}$ in a period interval. Since we are searching for the existence of periodic solutions for (1.2), it is natural to assume that

$$
q(t+T)=q(t), p(t+T)=p(t), a(t+T)=a(t), b(t+T)=b(t), r(t+T)=r(t) .
$$

Function $g(x)$ is globally Lipschitz continuous. That is, there is a positive constant $k$ such that

$$
|g(x)-g(y)| \leq k\|x-y\|_{0}
$$


we also assume that $g$ is continuously differentiable with $\left\|g^{\prime}\right\|_{0}=l_{1}$.

The next two lemmas will be used in the sequel.

Lemma 2.1. If $\mathrm{a}(\mathrm{t}) \neq 0, \mathrm{f} \in \mathrm{C}_{\boldsymbol{\omega}}$, then the scalar equation $\mathrm{x}^{\prime}(\mathrm{t})+\mathrm{a}(\mathrm{t}) \mathrm{x}(\mathrm{t})=\mathrm{f}(\mathrm{t})$ has a unique $\omega$-periodic solution

$$
x(t)=\left(1-e^{-\int_{t}^{t+\omega} a(u) d u}\right)^{-1} \int_{t}^{t+w} e^{\int_{t+w}^{s} a(u) d u} f(s) d s .
$$

Proof. It is easy to prove. We can find it in many ODE textbooks.

Lemma 2.2 ([27]). Let $\mathcal{M}$ be a closed convex nonempty subset of a Banach space $(\mathrm{S},\|\cdot\|)$. Suppose that $\mathrm{A}$ and $\mathrm{B}$ map $\mathcal{M}$ into $\mathrm{S}$ such that

(i) $x, y \in \mathcal{M}$, implies $A x+B y \in \mathcal{M}$,

(ii) $A$ is compact and continuous,

(iii) B is a contraction mapping.

Then there exists $z \in \mathcal{M}$ with $z=A z+B z$.

By applying Lemmas 2.1 and 2.2 we obtain in this section the existence of periodic solution of (1.2). For a sufficiently small positive $l,(1.2)$ can be transformed as

$$
\begin{aligned}
& \frac{d}{d t} v(t)-l q_{1}(t) \frac{d}{d t} g(v(t-\tau(t))) \\
& =l p_{1}(t)-l a_{1}(t) v(t)-l a_{1}(t) q_{1}(t) g(v(t-\tau(t)))-l b_{1}(t) f(v(t))+l b_{1}(t) q_{1}(t) f(v(t-\tau(t))),
\end{aligned}
$$

where $v(t)=u(l t), \tau(t)=\frac{r(l t)}{l}, q_{1}(t)=q(l t), p_{1}(t)=p(l t), a_{1}(t)=a(l t), b_{1}(t)=b(l t)$ and $\omega=\frac{\mathrm{T}}{\mathrm{l}}$.

Theorem 2.3. Suppose that $\mathrm{f} \in \mathrm{C}^{1}(\mathbb{R})$ and $\mathrm{q}_{1}, \mathrm{p}_{1}, \mathrm{a}_{1}, \mathrm{~b}_{1} \in \mathrm{C}_{\omega}^{1}$, we also assume that $\left\|\mathrm{q}_{1}^{\prime}\right\|=\beta$, and $\left\|1-\tau^{\prime}(\mathrm{t})\right\|=\mathrm{l}_{2}$, if there exist constants $\rho \in(0,1)$ and $\mathrm{H}>0$ such that

$$
\begin{gathered}
l k\left(\left\|q_{1}\right\|_{0}\left(1+l_{2} H\right)+\beta\right) \leq \rho \\
\sup _{|\mathfrak{u}| \leq H}|f(u)|<\frac{\theta_{1} H-\theta_{3}}{\left\|b_{1}\right\|_{0}},\left\|q_{1}\right\|_{0}<\frac{\theta_{1}-\frac{\theta_{3}+\left\|b_{1}\right\|_{\mathcal{O}} \sup _{|\mathfrak{u}| \leq H}|f(u)|}{H}}{\theta_{2}+\frac{\left\|b_{1}\right\|_{\mathcal{O}} \sup _{|\mathfrak{u}| \leq H}|f(u)|}{H}}
\end{gathered}
$$

and

$$
\left\|p_{1}\right\|_{0}<\left(\theta_{1}-\theta_{2}\left\|q_{1}\right\|_{0}\right) H-\left\|b_{1}\right\|_{0}\left(1+\left\|q_{1}\right\|_{0}\right) \sup _{|u| \leq H}|f(u)|-\theta_{3}
$$

where

$$
\begin{aligned}
\theta_{1} & =\frac{\frac{1}{l}-k \beta}{1+\alpha \omega M\left(1+l\left\|a_{1}\right\|_{0}\right)}, \theta_{2}=\frac{k+l_{1} l_{2}}{1+\alpha \omega M\left(1+l\left\|a_{1}\right\|_{0}\right)}+2 k\left\|a_{2}\right\|_{0}, \\
\theta_{3} & =\frac{\left(\left\|q_{1}\right\|_{0}+\beta\right)|g(0)|}{1+\alpha \omega M\left(1+l\left\|a_{1}\right\|_{0}\right)}+2\left\|a_{2}\right\|_{0}\left\|q_{1}\right\|_{0}|g(0)|, \\
2 a_{2}(s) q_{1}(s) & =(l+1) a_{1}(s) q_{1}(s)+q_{1}^{\prime}(s),
\end{aligned}
$$


and

$$
M=\sup _{t \in \mathbb{R}}\left|\int_{t}^{t+\omega} e^{\int_{t+\omega}^{s} l a_{1}(u) d u} d s\right|, \alpha=\left(1-e^{-\int_{t}^{t+w} l a_{1}(u) d u}\right)^{-1} .
$$

Then (1.2) has a T-periodic solution.

Proof. According to the conditions (2.2) and (2.3) we get

$$
\begin{aligned}
& \left(1+\alpha \omega M\left(1+l\left\|a_{1}\right\|_{0}\right)\right)\left[l\left\|p_{1}\right\|_{0}+l\left\|b_{1}\right\|_{0}\left(1+\left\|q_{1}\right\|_{0}\right) \sup _{|u| \leq H}|f(u)|\right. \\
& \left.+2 l\left\|a_{2}\right\|_{0}\left\|q_{1}\right\|_{0}(k H+|g(0)|)\right]+l\left(\left(k+l_{1} l_{2}\right)\left\|q_{1}\right\|_{0}+k \beta\right) H \\
& +l\left(\left\|q_{1}\right\|_{0}+\beta\right)|g(0)| \leq H
\end{aligned}
$$

We need to prove that (2.1) has a $\omega$-periodic solution. Let

$$
S=\left\{\phi \in C^{1}(\mathbb{R}),\|\phi\|_{1}=\|\phi\|_{0}+\left\|\phi^{\prime}\right\|_{0}<+\infty\right\},
$$

and

$$
\mathcal{M}=\left\{\phi \in \mathrm{C}_{\omega}^{1},\|\phi\|_{1} \leq \mathrm{H}\right\}
$$

then $\mathcal{M}$ is a bounded closed convex set of the Banach space $\mathrm{S}$.

For all $\phi \in \mathcal{M}$, consider the nonhomogeneous equation

$$
\begin{aligned}
& \frac{d}{d t} v(t)+l a_{1}(t) v(t) \\
& =\operatorname{lp}_{1}(t)-l a_{1}(t) q_{1}(t) g(v(t-\tau(t)))-l b_{1}(t) f(v(t)) \\
& +l b_{1}(t) q_{1}(t) f(v(t-\tau(t)))+l q_{1}(t) \frac{d}{d t} g(v(t-\tau(t))) .
\end{aligned}
$$

According to the Lemma 2.1 this equation has a unique $\omega$-periodic solution

$$
\begin{aligned}
v(t) & =\left(1-e^{-\int_{t}^{t+\omega} l a_{1}(u) d u}\right)^{-1} \int_{t}^{t+\omega} e^{\int_{t+\omega}^{s} l a_{1}(u) d u}\left[\operatorname{lp}_{1}(s)\right. \\
& -l a_{1}(s) q_{1}(s) g(v(s-\tau(s)))-l b_{1}(s) f(v(s)) \\
& \left.+l b_{1}(s) q_{1}(s) f(v(s-\tau(s)))+l q_{1}(s) \frac{d}{d s} g(v(s-\tau(s)))\right] d s
\end{aligned}
$$

Performing an integration by part, we obtain

$$
\begin{aligned}
v(t) & =\left(1-e^{-\int_{t}^{t+\omega} l a_{1}(u) d u}\right)^{-1} \int_{t}^{t+\omega} e^{\int_{t+\omega}^{s} l a_{1}(u) d u}\left[\operatorname{lp}_{1}(s)\right. \\
& -l\left((l+1) a_{1}(s) q_{1}(s)+q_{1}^{\prime}(s)\right) g(v(s-\tau(s)))-l b_{1}(s) f(v(s)) \\
& \left.+l b_{1}(s) q_{1}(s) f(v(s-\tau(s)))\right] d s+l q_{1}(t) g(v(t-\tau(t))),
\end{aligned}
$$

where $2 a_{2}(s) q_{1}(s)=(l+1) a_{1}(s) q_{1}(s)+q_{1}^{\prime}(s)$. Then

$$
\begin{aligned}
v(t) & =\left(1-e^{-\int_{t}^{t+\omega} l a_{1}(u) d u}\right)^{-1} \int_{t}^{t+w} e^{\int_{t+\omega}^{s} l a_{1}(u) d u}\left[\operatorname{lp}_{1}(s)\right. \\
& -2 l a_{2}(s) q_{1}(s) g(v(s-\tau(s)))-l b_{1}(s) f(v(s)) \\
& \left.+l b_{1}(s) q_{1}(s) f(v(s-\tau(s)))\right] d s+l q_{1}(t) g(v(t-\tau(t))) .
\end{aligned}
$$


Define operators A and B by

$$
\begin{aligned}
(A \phi)(t) & =\left(1-e^{-\int_{t}^{t+\omega} l a_{1}(u) d u}\right)^{-1} \int_{t}^{t+\omega} e^{\int_{t+\omega}^{s} l a_{1}(u) d u}\left[\operatorname{lp}_{1}(s)\right. \\
& -2 l a_{2}(s) q_{1}(s) g(\phi(s-\tau(s)))-l b_{1}(s) f(\phi(s)) \\
& \left.+l b_{1}(s) q_{1}(s) f(\phi(s-\tau(s)))\right] d s
\end{aligned}
$$

and

$$
(B \phi)(t)=l q_{1}(t) g(\phi(t-\tau(t))) .
$$

In order to prove that (2.1) has a periodic solution, we shall make sure that $A$ and $B$ satisfy the conditions of lemma 2.2. For all $x, y \in \mathcal{M}$, we have $x(t+w)=x(t), y(t+w)=y(t)$ and $\|x\|_{1} \leq H$, $\|y\|_{1} \leq \mathrm{H}$. Now let us discuss $A x+$ By.

$$
\begin{aligned}
(A x)(t+w) & =\left(1-e^{-\int_{t+\omega}^{t+2 \omega} l a_{1}(u) d u}\right)^{-1} \int_{t+\omega}^{t+2 \omega} e^{\int_{t+2 \omega}^{s} l a_{1}(u) d u}\left[\operatorname{lp}_{1}(s)\right. \\
& -2 l a_{2}(s) q_{1}(s) g(x(s-\tau(s)))-l b_{1}(s) f(x(s)) \\
& \left.+l b_{1}(s) q_{1}(s) f(x(s-\tau(s)))\right] d s \\
& =\left(1-e^{-\int_{t}^{t+\omega} l a_{1}(u) d u}\right)^{-1} \int_{t}^{t+\omega} e^{\int_{t+\omega}^{s} l a_{1}(u) d u}\left[\operatorname{lp}_{1}(s)\right. \\
& -2 l a_{2}(s) q_{1}(s) g(x(s-\tau(s)))-l b_{1}(s) f(x(s) \\
& \left.+l b_{1}(s) q_{1}(s) f(x(s-\tau(s)))\right] d s \\
& =(A x)(t)
\end{aligned}
$$

and

$$
\begin{aligned}
(B y)(t+\omega) & =l_{1}(t+\omega) g(y(t+\omega-\tau(t+\omega))) \\
& =\operatorname{lq}_{1}(t) g(y(t-\tau(t))=(B y)(t),
\end{aligned}
$$

therefore $(A x+B y)(t+w)=(A x+B y)(t)$. Meanwhile, we get

$$
\begin{aligned}
(A x)^{\prime}(t) & =-l a_{1}(t)\left(1-e^{-\int_{t}^{t+\omega} l a_{1}(u) d u}\right)^{-1} \int_{t}^{t+w} e^{\int_{t+\omega}^{s} l a_{1}(u) d u}\left[\operatorname{lp}_{1}(s)\right. \\
& -2 l a_{2}(s) q_{1}(s) g(x(s-\tau(s)))-l b_{1}(s) f(x(s)) \\
& \left.+l b_{1}(s) q_{1}(s) f(x(s-\tau(s)))\right] d s \\
& +\left[\operatorname{lp}_{1}(t)-2 l a_{2}(t) q_{1}(t) g(x(t-\tau(t)))-l b_{1}(t) f(x(t))\right. \\
& \left.+l b_{1}(t) q_{1}(t) f(x(t-\tau(t)))\right]
\end{aligned}
$$

and

$$
(B y)^{\prime}(t)=l q_{1}^{\prime}(t) g(y(t-\tau(t)))+l q_{1}(t)\left(1-\tau^{\prime}(t)\right) y^{\prime}(t-\tau(t)) g^{\prime}(y(t-\tau(t)))
$$


Thus,

$$
\begin{aligned}
\|A x\|_{1} & =\|A x\|_{0}+\left\|(A x)^{\prime}\right\|_{0} \\
& \leq\left(1+\alpha \omega M\left(1+l\left\|a_{1}\right\|_{0}\right)\right)\left[l\left\|p_{1}\right\|_{0}+l\left\|b_{1}\right\|_{0}\left(1+\left\|q_{1}\right\|_{0}\right) \sup _{|u| \leq H}|f(u)|\right. \\
& \left.+2 l\left\|a_{2}\right\|_{0}\left\|q_{1}\right\|_{0}(k H+|g(0)|)\right],
\end{aligned}
$$

and

$$
\begin{aligned}
\|B y\|_{1} & =\|B y\|_{0}+\left\|(B y)^{\prime}\right\|_{0} \\
& \leq l\left(\left(k+l_{1} l_{2}\right)\left\|q_{1}\right\|_{0}+k \beta\right) H+l\left(\left\|q_{1}\right\|_{0}+\beta\right)|g(0)|,
\end{aligned}
$$

therefore

$$
\begin{aligned}
& \|A x+B y\|_{1} \\
& \leq\|A x\|_{1}+\|B y\|_{1} \\
& \leq\left(1+\alpha \omega M\left(1+l\left\|a_{1}\right\|_{0}\right)\right)\left[l\left\|p_{1}\right\|_{0}+l\left\|b_{1}\right\|_{0}\left(1+\left\|q_{1}\right\|_{0}\right) \sup _{|u| \leq H}|f(u)|\right. \\
& \left.+2 l\left\|a_{2}\right\|_{0}\left\|q_{1}\right\|_{0}(k H+|g(0)|)\right]+l\left(\left(k+l_{1} l_{2}\right)\left\|q_{1}\right\|_{0}+k \beta\right) H \\
& +l\left(\left\|q_{1}\right\|_{0}+\beta\right)|g(0)|,
\end{aligned}
$$

by (2.4), $\|A x+B y\|_{1} \leq \mathrm{H}$. Accordingly, $A x+B y \in \mathcal{M}$.

For all $x \in \mathcal{M},\|A x\|_{0} \leq \mathrm{H},\left\|(A x)^{\prime}\right\|_{0} \leq \mathrm{H}$. According to Ascoli-Arzila lemma, the subset $A \mathcal{M}$ of $\mathbf{C}_{\omega}$ is a precompact set, therefore for all sequence $\left\{x_{n}\right\}$ of $\mathcal{M}$, there exists the subsequence $\left\{x_{n_{k}}\right\}$ of $\left\{x_{n}\right\}$ such that $A x_{n_{k}} \rightarrow x_{0} \in \mathbf{C}_{\omega}$ as $k \rightarrow+\infty$. Meanwhile, we get,

$$
\begin{aligned}
(A x)^{\prime \prime}(t) & =\left(l^{2} a_{1}^{2}(t)-l a_{1}^{\prime}(t)\right)\left(1-e^{-\int_{t}^{t+w} l a_{1}(u) d u}\right)^{-1} \int_{t}^{t+w} e^{\int_{t+w}^{s} l a_{1}(u) d u}\left[p_{1}(s)\right. \\
& -2 l a_{2}(s) q_{1}(s) g(x(s-\tau(s)))-l b_{1}(s) f(x(s)) \\
& \left.+l b_{1}(s) q_{1}(s) f(x(s-\tau(s)))\right] d s \\
& -l a_{1}(t)\left[l p_{1}(t)-2 l a_{2}(t) q_{1}(t) g(x(t-\tau(t)))-l b_{1}(t) f(x(t))\right. \\
& \left.+l b_{1}(t) q_{1}(t) f(x(t-\tau(t)))\right] \\
& +\left[l p_{1}^{\prime}(t)-2 l a_{2}^{\prime}(t) q_{1}(t) g(x(t-\tau(t)))-2 l a_{2}(t) q_{1}^{\prime}(t) g(x(t-\tau(t)))\right. \\
& -2 l a_{2}(t) q_{1}(t)\left(1-\tau^{\prime}(t)\right) x^{\prime}(t-\tau(t)) g^{\prime}(x(t-\tau(t))) \\
& -l b_{1}^{\prime}(t) f(x(t))-l b_{1}(t) x^{\prime}(t) f^{\prime}(x(t))+l b_{1}^{\prime}(t) q_{1}(t) f(x(t-\tau(t))) \\
& +l b_{1}(t) q_{1}^{\prime}(t) f(x(t-\tau(t))) \\
& \left.+l b_{1}(t) q_{1}(t)\left(1-\tau^{\prime}(t)\right) x^{\prime}(t-\tau(t)) f^{\prime}(x(t-\tau(t)))\right] .
\end{aligned}
$$


Thus

$$
\begin{aligned}
& \sup _{\mathfrak{t} \in \mathbb{R}}\left|(A x)^{\prime \prime}(\mathrm{t})\right| \\
& \leq\left[\left(l^{2}\left\|a_{1}^{2}\right\|_{0}+l\left\|a_{1}^{\prime}\right\|_{0}\right) \alpha \omega M+l\left\|a_{1}\right\|_{0}\right]\left[l\left\|p_{1}\right\|_{0}\right. \\
& \left.+l\left\|b_{1}\right\|_{0}\left(1+\left\|q_{1}\right\|_{0}\right) \sup _{|u| \leq H}|f(u)|+2 l\left\|a_{2}\right\|_{0}\left\|q_{1}\right\|_{0}(k H+|g(0)|)\right] \\
& +l\left\|p_{1}^{\prime}\right\|_{0}+l\left(\left\|b_{1}^{\prime}\right\|_{0}\left(1+\left\|q_{1}\right\|_{0}\right)+\beta\left\|b_{1}\right\|_{0}\right) \sup _{|u| \leq H}|f(u)| \\
& +l\left(\left\|b_{1}\right\|\left\|f^{\prime}\right\|_{0}\left(1+l_{2}\left\|q_{1}\right\|_{0}\right)+2 l_{1} l_{2}\left\|a_{2}\right\|_{0}\left\|q_{1}\right\|_{0}\right) H \\
& +2 l\left(\left\|a_{2}^{\prime}\right\|_{0}\left\|q_{1}\right\|_{0}+\beta\left\|a_{2}\right\|_{0}\right)(k H+|g(0)|) .
\end{aligned}
$$

Therefore there is a constant $H_{1}>0$ such that $\sup _{t \in \mathbb{R}}\left|(A x)^{\prime \prime}(t)\right| \leq H_{1}$ and $\left\{(A x)^{\prime}: x \in \mathcal{M}\right\} \subset C_{\omega}$. According to Ascoli-Arzela lemma, for all sequence $\left\{x_{n}\right\}$ of $\mathcal{M}$, there exists the subsequence $\left\{x_{n_{k}}\right\}$ of $\left\{x_{n}\right\}$, such that $\left(A x_{n_{k}}\right)^{\prime} \rightarrow z_{0} \in C_{\omega}$. Since $\frac{d}{d t}$ is a closed operator, $z_{0}=\left(x_{0}\right)^{\prime}$. Hence, $x_{0} \in C_{\omega}^{1}$ and $\left\{A x_{n}\right\}$ is contained in a compact set. $A$ is a compact operator.

Suppose that $\left\{x_{n}\right\} \subset \mathcal{M}, x \in S, x_{n} \rightarrow x$, then $\left\|x_{n}-x\right\|_{0} \rightarrow 0$ and $\left\|x_{n}^{\prime}-x^{\prime}\right\|_{0} \rightarrow 0$ as $n \rightarrow \infty$, and we get

$$
\begin{aligned}
& \left\|A x_{n}-A x\right\|_{0} \\
& \leq \alpha \omega M\left[2 l\left\|a_{2}\right\|_{0}\left\|q_{1}\right\|_{0} k\left\|x_{n}(t)-x(t)\right\|_{0}\right. \\
& \left.+l\left\|b_{1}\right\|_{0}\left(1+\left\|q_{1}\right\|_{0}\right) \sup _{t \in[0, \omega]}\left|f\left(x_{n}(t)\right)-f(x(t))\right|\right]
\end{aligned}
$$

and

$$
\begin{aligned}
& \left\|\left(A x_{n}\right)^{\prime}-(A x)^{\prime}\right\|_{0} \\
& \leq\left(l \alpha \omega M\left\|a_{1}\right\|_{0}+1\right)\left[2 l\left\|a_{2}\right\|_{0}\left\|q_{1}\right\|_{0} k\left\|x_{n}(t)-x(t)\right\|_{0}\right. \\
& \left.+l\left\|b_{1}\right\|_{0}\left(1+\left\|q_{1}\right\|_{0}\right) \sup _{t \in[0, \omega]}\left|f\left(x_{n}(t)\right)-f(x(t))\right|\right],
\end{aligned}
$$

when $\left\|x_{n}-x\right\|_{1} \rightarrow 0$ as $n \rightarrow \infty$ and $\left\|x_{n}(t)-x(t)\right\|_{0} \rightarrow 0$ for $t \in[0, \omega]$ uniformly. And since $f$ is continuous, $\left\|A x_{n}-A x\right\|_{0} \rightarrow 0,\left\|\left(A x_{n}\right)^{\prime}-(A x)^{\prime}\right\|_{0} \rightarrow 0$. Consequently, $A$ is continuous.

For all $x, y \in \mathcal{M}$

$$
\begin{aligned}
& \|B x-B y\|_{1} \\
& =\|B x-B y\|_{0}+\left\|(B x)^{\prime}-(B y)^{\prime}\right\|_{0} \\
& \leq l k\left(\left\|q_{1}\right\|_{0}\left(1+l_{2} H\right)+\beta\right)\|x-y\|_{0} \leq \rho\|x-y\|_{0} .
\end{aligned}
$$

Therefore B is a contraction operator.

Thus, the conditions of Lemma 2.2 are satisfied and there is a $\phi \in \mathcal{M}$, such that $\phi=A \phi+B \phi$. It is $\omega$-periodic solution for (2.1). Since $v(t)=u(l t), p_{1}(t)=p(l t), q_{1}(t)=q(l t), b_{1}(t)=b(l t)$ and $a_{1}(t)=a(l t),(1.2)$ has a T-periodic solution. 


\section{Asymptotic stability of periodic solutions}

This section concerned with the asymptotic stability of periodic solutions. Let $u^{*}$ is the equilibrium of (1.2). Let $v=u-u^{*}$ then (1.2) is transformed as

$$
\begin{aligned}
& \frac{d}{d t} v(t)-q(t) \frac{d}{d t}\left(g\left(v(t-r(t))+u^{*}(t-r(t))\right)-g\left(u^{*}(t-r(t))\right)\right) \\
& =-a(t) v(t)-a(t) q(t)\left(g\left(v(t-r(t))+u^{*}(t-r(t))\right)-g\left(u^{*}(t-r(t))\right)\right) \\
& -b(t)\left(f\left(v(t)+u^{*}(t)\right)-f\left(u^{*}(t)\right)\right) \\
& +b(t) q(t)\left(f\left(v(t-r(t))+u^{*}(t-r(t))\right)-f\left(u^{*}(t-r(t))\right)\right) .
\end{aligned}
$$

Obviously (3.1) has the zero solution. Now we use Krasnoselskii's fixed point theorem to prove that the zero solution of (3.1) is asymptotically stable. We set $S$ as the Banach space of bounded continuous function $\phi:[\mathrm{m}(0), \infty) \rightarrow \mathbb{R}$ with the supremum norm $\|\cdot\|$ and $m(0)=\inf \{t-r(t)$, $t \geq 0\}$. Also, given the initial function $\psi$, denote the norm of $\psi$ by $\|\psi\|=\sup _{t \in[m(0), 0]}|\psi(t)|$, which should not cause confusion with the same symbol for the norm in $\mathrm{S}$.

Theorem 3.1. If all conditions of Theorem 2.3 are satisfies, $f$ satisfies the locally Lipschitz condition. Further assume that

$$
\int_{0}^{t} a(u) d u>0, e^{-\int_{0}^{t} a(u) d u} \rightarrow 0, t-r(t) \rightarrow \infty \text { as } t \rightarrow \infty
$$

and

$$
k\|\mathrm{q}\|<1,
$$

and there exists $\mathrm{R}>\mathrm{H}$ such that

$$
\|\mathbf{b}\| \sup _{|\mathfrak{u}| \leq \mathrm{H}+\mathrm{R}}|\mathrm{f}(\mathrm{u})|<\left(\frac{1}{\delta}-\mathrm{k} \beta\right) \mathrm{R}-\left(2 \mathrm{k} \beta+\theta_{1}\right) \mathrm{H}-\theta_{3},
$$

and that

$$
\|\mathrm{q}\|<\frac{(1-k \delta \beta) \mathrm{R}-2 \mathrm{k} \delta \beta \mathrm{H}-\delta\|\mathrm{b}\| \sup _{|\mathfrak{u}| \leq \mathrm{H}+\mathrm{R}}|\mathrm{f}(\mathrm{u})|-\delta\left(\theta_{1} \mathrm{H}+\theta_{3}\right)}{(2 \delta\|\mathrm{a}\|+1) \mathrm{kR}+4 \mathrm{k} \delta\|\mathrm{a}\| \mathrm{H}+\delta\|\mathrm{b}\| \sup _{|\mathfrak{u}| \leq \mathrm{H}+\mathrm{R}}|\mathrm{f}(\mathrm{u})|+\delta\left(\theta_{1} \mathrm{H}+\theta_{3}\right)}
$$

and

$$
\begin{aligned}
\|\psi\| & \leq\{R-(k \delta(2\|a\|\|q\|+\beta)+k\|q\|) R \\
& -2 k \delta(2\|a\|\|q\|+\beta) H-\delta\|b\|(1+\|q\|) \sup _{|u| \leq H+R}|f(u)| \\
& \left.-\delta(1+\|q\|)\left(\theta_{1} H+\theta_{3}\right)\right\} /(1+k\|q\|),
\end{aligned}
$$

where

$$
\sup _{t \geq 0}\left|\int_{0}^{t} e^{f_{-s}^{t} a(u) d u} d s\right| \leq \delta
$$

Then the solution of (3.1) $v(t) \rightarrow 0$ as $t \rightarrow \infty$. 
Proof. According to the conditions (3.2), (3.3) and (3.4), we have

$$
\begin{aligned}
& 2 k \delta(2\|a\|\|q\|+\beta) H+[k \delta(2\|a\|\|q\|+\beta)+k\|q\|] R \\
& +\delta\|b\|(1+\|q\|) \sup _{|u| \leq H+R}|f(u)|+\delta(1+\|q\|)\left(\theta_{1} H+\theta_{3}\right) \\
& +(1+k\|q\|)\|\psi\| \leq R .
\end{aligned}
$$

Given the initial function $\psi$, there exists a unique solution $v$ for (3.1). Let

$$
\mathcal{M}_{\psi}=\{\phi \in S,\|\phi\| \leq R, \phi(t)=\psi(t) \text { for } \mathrm{t} \in[\mathrm{m}(0), 0],|\phi(t)| \rightarrow 0 \text { as } \mathrm{t} \rightarrow \infty\},
$$

then $\mathcal{M}_{\Psi}$ is a bounded convex closed set of $\mathrm{S}$. We write (3.1) as

$$
\begin{aligned}
& \frac{d}{d t} v(t)+a(t) v(t) \\
& =-a(t) q(t)\left(g\left(v(t-r(t))+u^{*}(t-r(t))\right)-g\left(u^{*}(t-r(t))\right)\right) \\
& +q(t) \frac{d}{d t}\left(g\left(v(t-r(t))+u^{*}(t-r(t))\right)-g\left(u^{*}(t-r(t))\right)\right) \\
& -b(t)\left(f\left(v(t)+u^{*}(t)\right)-f\left(u^{*}(t)\right)\right) \\
& +b(t) q(t)\left(f\left(v(t-r(t))+u^{*}(t-r(t))\right)-f\left(u^{*}(t-r(t))\right)\right)
\end{aligned}
$$

According to Lemma 2.1 this equation has a unique solution written as

$$
\begin{aligned}
v(t) & =v(0) e^{-\int_{0}^{t} a(u) d u} \\
& +\int_{0}^{t} e^{-\int_{s}^{t} a(u) d u}\left[-a(s) q(s)\left(g\left(v(s-r(s))+u^{*}(s-r(s))\right)-g\left(u^{*}(s-r(s))\right)\right)\right. \\
& +q(t) \frac{d}{d s}\left(g\left(v(s-r(s))+u^{*}(s-r(s))\right)-g\left(u^{*}(s-r(s))\right)\right) \\
& -b(s)\left(f\left(v(s)+u^{*}(s)\right)-f\left(u^{*}(s)\right)\right) \\
& \left.+b(s) q(s)\left(f\left(v(s-r(s))+u^{*}(s-r(s))\right)-f\left(u^{*}(s-r(s))\right)\right)\right] d s .
\end{aligned}
$$

Performing an integration by part, we obtain

$$
\begin{aligned}
v(t) & =\left(v(0)-q(0)\left(g\left(v(-r(0))+u^{*}(-r(0))\right)-g\left(u^{*}(-r(0))\right)\right) e^{-\int_{0}^{t} a(u) d u}\right. \\
& +q(t)\left(g\left(v(t-r(t))+u^{*}(t-r(t))\right)-g\left(u^{*}(t-r(t))\right)\right) \\
& +\int_{0}^{t} e^{-\int_{s}^{t} a(u) d u}\left[-\left(2 a(s) q(s)+q^{\prime}(s)\right)\right. \\
& \times\left(g\left(v(s-r(s))+u^{*}(s-r(s))\right)-g\left(u^{*}(s-r(s))\right)\right) \\
& -b(s)\left(f\left(v(s)+u^{*}(s)\right)-f\left(u^{*}(s)\right)\right) \\
& \left.+b(s) q(s)\left(f\left(v(s-r(s))+u^{*}(s-r(s))\right)-f\left(u^{*}(s-r(s))\right)\right)\right] d s .
\end{aligned}
$$


Let $v(t)=\psi(t), t \in[m(0), 0]$, and

$$
\begin{aligned}
(L \phi)(t) & =\left(\psi(0)-q(0)\left(g\left(\psi(-r(0))+u^{*}(-r(0))\right)-g\left(u^{*}(-r(0))\right)\right) e^{-\int_{0}^{t} a(u) d u}\right. \\
& +q(t)\left(g\left(\phi(t-r(t))+u^{*}(t-r(t))\right)-g\left(u^{*}(t-r(t))\right)\right) \\
& +\int_{0}^{t} e^{-\int_{s}^{t} a(u) d u}\left[-\left(2 a(s) q(s)+q^{\prime}(s)\right)\right. \\
& \times\left(g\left(\phi(s-r(s))+u^{*}(s-r(s))\right)-g\left(u^{*}(s-r(s))\right)\right) \\
& -b(s)\left(f\left(\phi(s)+u^{*}(s)\right)-f\left(u^{*}(s)\right)\right) \\
& \left.+b(s) q(s)\left(f\left(\phi(s-r(s))+u^{*}(s-r(s))\right)-f\left(u^{*}(s-r(s))\right)\right)\right] d s
\end{aligned}
$$

For all $\phi \in \mathcal{M}_{\psi}$, define the operators $A$ and $B$ by

$$
\begin{gathered}
(A \phi)(t)=\left\{\begin{array}{l}
0, t \in[m(0), 0] \\
\int_{0}^{t} e^{-\int_{s}^{t} a(u) d u}\left[-\left(2 a(s) q(s)+q^{\prime}(s)\right)\right. \\
\times\left(g\left(\phi(s-r(s))+u^{*}(s-r(s))\right)-g\left(u^{*}(s-r(s))\right)\right) \\
-b(s)\left(f\left(\phi(s)+u^{*}(s)\right)-f\left(u^{*}(s)\right)\right) \\
+b(s) q(s)\left(f\left(\phi(s-r(s))+u^{*}(s-r(s))\right)\right] d s, t \geq 0
\end{array}\right. \\
(B \phi)(t)=\left\{\begin{array}{l}
\psi(t), t \in[m(0), 0], \\
\left(\psi(0)-q(0)\left(g\left(\psi(-r(0))+u^{*}(-r(0))\right)-g\left(u^{*}(-r(0))\right)\right) e^{-\int_{0}^{t} a(u) d u}\right. \\
+q(t)\left(g\left(\phi(t-r(t))+u^{*}(t-r(t))\right)-g\left(u^{*}(t-r(t))\right)\right), t \geq 0
\end{array}\right.
\end{gathered}
$$

(i) For all $x, y \in \mathcal{M}_{\psi}, x(t) \rightarrow 0$ and $y(t) \rightarrow 0$ as $t \rightarrow \infty$ then

$$
\begin{aligned}
(B y)(t) & =\left(\psi(0)-q(0)\left(g\left(\psi(-r(0))+u^{*}(-r(0))\right)-g\left(u^{*}(-r(0))\right)\right) e^{-\int_{0}^{t} a(u) d u}\right. \\
& +q(t)\left(g\left(y(t-r(t))+u^{*}(t-r(t))-g\left(u^{*}(t-r(t))\right)\right) \rightarrow 0 \text { as } t \rightarrow \infty\right.
\end{aligned}
$$

and

$$
\begin{aligned}
& \lim _{t \rightarrow \infty}(A x)(t) \\
& =\lim _{t \rightarrow \infty}\left\{\int _ { 0 } ^ { t } e ^ { \int _ { 0 } ^ { s } a ( u ) d u } \left[-\left(2 a(s) q(s)+q^{\prime}(s)\right)\right.\right. \\
& \times\left(g\left(x(s-r(s))+u^{*}(s-r(s))\right)-g\left(u^{*}(s-r(s))\right)\right) \\
& -b(s)\left(f\left(x(s)+u^{*}(s)\right)-f\left(u^{*}(s)\right)\right) \\
& \left.\left.+b(s) q(s)\left(f\left(x(s-r(s))+u^{*}(s-r(s))\right)-f\left(u^{*}(s-r(s))\right)\right)\right] d s\right\} / e^{\int_{0}^{t} a(u) d u} \\
& =0,
\end{aligned}
$$


therefore $\lim _{t \rightarrow \infty}(A x+B y)(t)=0$, and

$$
\begin{aligned}
\|A x\| & \leq\left\{\left(2\|\mathrm{a}\|\|\mathrm{q}\|+\left\|\mathrm{q}^{\prime}\right\|\right)\left[\sup _{|x| \leq \mathrm{H}+\mathrm{R}}|\mathrm{g}(\mathrm{x})|+\sup _{|x| \leq \mathrm{H}}|\mathrm{g}(\mathrm{x})|\right]\right. \\
& \left.+\|\mathrm{b}\|(1+\|\mathrm{q}\|)\left[\sup _{|x| \leq \mathrm{H}+\mathrm{R}}|\mathrm{f}(\mathrm{x})|+\sup _{|x| \leq \mathrm{H}} \mid \mathrm{f}(\mathrm{x})\right]\right\} \sup _{\mathrm{t} \geq 0}\left|\int_{0}^{\mathrm{t}} e^{-\int_{\mathrm{s}}^{\mathrm{t}} \mathrm{a}(\mathrm{u}) \mathrm{du}} \mathrm{ds}\right| \\
& \leq 2 \mathrm{k} \delta(2\|\mathrm{a}\|\|\mathrm{q}\|+\beta) \mathrm{H}+\mathrm{k} \delta(2\|\mathrm{a}\|\|\mathrm{q}\|+\beta) \mathrm{R} \\
& +\delta\|\mathrm{b}\|(1+\|\mathrm{q}\|) \sup _{|x| \leq \mathrm{H}+\mathrm{R}}|\mathrm{f}(\mathrm{x})|+\delta(1+\|\mathrm{q}\|)\left(\theta_{1} \mathrm{H}+\theta_{3}\right),
\end{aligned}
$$

and

$$
\begin{aligned}
\|B y\| & =\sup _{t \geq m(0)}|(B y)(t)| \\
& =\max \left\{\|\psi\|, \sup _{t \geq 0} \mid\left(\psi(0)-q(0)\left(g\left(\psi(-r(0))+u^{*}(-r(0))\right)-g\left(u^{*}(-r(0))\right)\right) e^{-\int_{0}^{t} a(u) d u}\right.\right. \\
& +q(t)\left(g\left(y(t-r(t))+u^{*}(t-r(t))-g\left(u^{*}(t-r(t))\right)\right) \mid\right\} \\
& \leq(1+k\|q\|)\|\psi\|+k\|q\| R .
\end{aligned}
$$

Thus

$$
\begin{aligned}
& \|A x+B y\| \\
& \leq\|A x\|+\|B y\| \\
& \leq 2 k \delta(2\|a\|\|q\|+\beta) H+[k \delta(2\|a\|\|q\|+\beta)+k\|q\|] R \\
& +\delta\|b\|(1+\|q\|) \sup _{|x| \leq H+R}|f(x)|+\delta(1+\|q\|)\left(\theta_{1} H+\theta_{3}\right)+(1+k\|q\|)\|\psi\| .
\end{aligned}
$$

According to the condition (3.5), $\|A x+B y\| \leq R$. Thus $A x+B y \in \mathcal{M}_{\psi}$.

(ii) For all $x \in \mathcal{M}_{\psi},\|x\| \leq R,\left|(A x)^{\prime}(t)\right|=0, t \in[m(0), 0]$,

$$
\begin{aligned}
\left|(A x)^{\prime}(\mathrm{t})\right| & \leq(1+\|\mathrm{a}\| \delta)[k(2\|\mathrm{a}\|\|\mathrm{q}\|+\beta)(2 \mathrm{H}+\mathrm{R}) \\
& \left.+\|\mathrm{b}\|(1+\|\mathrm{q}\|) \sup _{|x| \leq \mathrm{H}+\mathrm{R}}|\mathrm{f}(\mathrm{x})|+(1+\|\mathrm{q}\|)\left(\theta_{1} \mathrm{H}+\theta_{3}\right)\right],
\end{aligned}
$$

here, the derivative of $(A x)^{\prime}(t)$ at zero means the left hand side derivative when $t \in[m(0), 0]$ and the right hand side derivative when $t \geq 0$. We can see $\left|(A x)^{\prime}(t)\right|$ is bounded for all $\psi \in \mathcal{M}_{\psi}$ and $A \mathcal{M}_{\psi}$ is a precompact set of $S$. Therefore $A$ is compact.

Let $\left(x_{n}\right) \subset \mathcal{M}_{\psi}, x \in S, x_{n} \rightarrow x$ as $n \rightarrow \infty$, then $\left|x_{n}(t)-x(t)\right| \rightarrow 0$ uniformly for $t \geq m(0)$ as $\mathrm{n} \rightarrow \infty$. Since

$$
\begin{aligned}
& \left\|A x_{n}-A x\right\| \\
& \leq \delta\left[k(2\|a\|\|q\|+\beta)\left\|x_{n}-x\right\|+k_{1}\|b\|(1+\|q\|)\left\|x_{n}-x\right\|\right],
\end{aligned}
$$

and $\mathrm{f}$ is continuous therefore, $\left\|A x_{n}-A x\right\| \rightarrow 0$ as $n \rightarrow \infty$ and $A$ is continuous. 
(iii) For all $x, y \in \mathcal{M}_{\psi}$,

$$
\|B x-B y\| \leq k\|q\|\|x-y\|,
$$

therefore B is a contraction operator.

According to Krasnoselskii's fixed point theorem, there is a $\phi \in \mathcal{M}_{\psi}$ such that $(A+B) \phi=\phi$ and $\phi$ is a solution for (3.1). Because the solution through $\psi$ for the equation is unique, the solution $v(t) \rightarrow 0$ as $t \rightarrow \infty$.

When $f$ and $g$ satisfy the locally Lipschitz condition, $H$ in Theorem 2.3 and $R$ in Theorem 3.1 exist, there are constant $P, k>0$ such that $\left|f\left(v(t)+u^{*}(t)\right)-f\left(u^{*}(t)\right)\right|<P|v(t)|$ and $\mid g(v(t)+$ $\left.u^{*}(t)\right)-g\left(u^{*}(t)\right)|<k| v(t) \mid$. Since $\phi$ satisfies

$$
\begin{aligned}
\phi(t) & =\left(\psi(0)-q(0)\left(g\left(\psi(-r(0))+u^{*}(-r(0))\right)-g\left(u^{*}(-r(0))\right)\right) e^{-\int_{0}^{t} a(u) d u}\right. \\
& +q(t)\left(g\left(\phi(t-r(t))+u^{*}(t-r(t))\right)-g\left(u^{*}(t-r(t))\right)\right) \\
& +\int_{0}^{t} e^{-\int_{s}^{t} a(u) d u}\left[-\left(2 a(s) q(s)+q^{\prime}(s)\right)\right. \\
& \times\left(g\left(\phi(s-r(s))+u^{*}(s-r(s))\right)-g\left(u^{*}(s-r(s))\right)\right) \\
& -b(s)\left(f\left(\phi(s)+u^{*}(s)\right)-f\left(u^{*}(s)\right)\right) \\
& \left.+b(s) q(s)\left(f\left(\phi(s-r(s))+u^{*}(s-r(s))\right)-f\left(u^{*}(s-r(s))\right)\right)\right] d s
\end{aligned}
$$

then

$$
\|\phi\| \leq(1+k\|q\|)\|\psi\|+k\|q\|\|\phi\|+\delta[k(2\|a\|\|q\|+\beta)\|\phi\|+\|b\|(1+\|q\|) P\|\phi\|]
$$

that is

$$
[1-k\|q\|-\delta k(2\|a\|\|q\|+\beta)-\delta\|b\|(1+\|q\|) P]\|\phi\| \leq(1+k\|q\|)\|\psi\| \text {. }
$$

Then there clearly exists a $\sigma>0$ for each $\epsilon>0$ such that $|\phi(t)|<\epsilon$ for all $t \geq m(0)$ if $\|\psi\|<\sigma$. Thus we have the following theorem.

Theorem 3.2. If $\mathrm{P}$ and $\mathrm{k}$ satisfy

$$
1-k\|q\|-\delta k(2\|a\|\|q\|+\beta)-\delta\|b\|(1+\|q\|) P>0
$$

Then the zero solution for (3.1) is stable.

\section{Conclusion}

In this paper, we provided the existence and asymptotic stability of periodic solutions with sufficient conditions for nonlinear neutral differential equations. The main tool of this paper is the method of fixed points. However, by introducing new fixed mappings, we get new existence and stability conditions. The obtained results have a contribution to the related literature, and they improve 
and extend the results in [19] from the cases of linear neutral term with constant delay to that general nonlinear cases with variable delay.

Acknowledgement. The authors would like to thank the anonymous referee for his/her valuable comments and good advice.

\section{References}

[1] A. Ardjouni and A. Djoudi, Existence of periodic solutions for a second-order nonlinear neutral differential equation with variable delay, Palestine Journal of Mathematics, Vol. 3(2) (2014), 191-197.

[2] A. Ardjouni, A. Djoudi and A. Rezaiguia, Existence of positive periodic solutions for two types of third-order nonlinear neutral differential equations with variable delay, Applied Mathematics E-Notes, 14 (2014), 86-96.

[3] A. Ardjouni and A. Djoudi, Existence of positive periodic solutions for a nonlinear neutral differential equations with variable delay, Applied Mathematics E-Notes, 12 (2012), 94-101.

[4] A. Ardjouni and A. Djoudi, Existence of periodic solutions for a second order nonlinear neutral differential equation with functional delay, Electronic Journal of Qualitative Theory of Differential Equations, 2012, No. 31, 1-9.

[5] A. Ardjouni and A. Djoudi, Periodic solutions for a second-order nonlinear neutral differential equation with variable delay, Electron. J. Differential Equations, Vol. 2011 (2011), No. 128, pp. 1-7.

[6] A. Ardjouni and A. Djoudi, Periodic solutions in totally nonlinear dynamic equations with functional delay on a time scale, Rend. Sem. Mat. Univ. Politec. Torino Vol. 68, 4(2010), 349-359.

[7] T. A. Burton, Liapunov functionals, fixed points and stability by Krasnoselskii's theorem, Nonlinear Stud. 9 (2002), No. 2, 181-190.

[8] T. A. Burton, Stability by Fixed Point Theory for Functional Differential Equations, Dover Publications, New York, 2006.

[9] T. Candan, Existence of positive periodic solutions of first-order neutral differential equations, Math. Methods Appl. Sci. 40 (2017), 205-209.

[10] T. Candan, Existence of positive periodic solutions of first-order neutral differential equations with variable coefficients, Applied Mathematics Letters 52 (2016), 142-148.

[11] F. D. Chen, Positive periodic solutions of neutral Lotka-Volterra system with feedback control, Appl. Math. Comput. 162 (2005), No. 3, 1279-1302. 
[12] F. D. Chen and J. L. Shi, Periodicity in a nonlinear predator-prey system with state dependent delays, Acta Math. Appl. Sin. Engl. Ser. 21 (2005), no. 1, 49-60.

[13] Z. Cheng and J. Ren, Existence of positive periodic solution for variable-coefficient third-order differential equation with singularity, Math. Meth. Appl. Sci. 2014, 37, 2281-2289.

[14] Z. Cheng and Y. Xin, Multiplicity Results for variable-coefficient singular third-order differential equation with a parameter, Abstract and Applied Analysis, Vol. 2014, Article ID 527162, $1-10$.

[15] S. Cheng and G. Zhang, Existence of positive periodic solutions for non-autonomous functional differential equations, Electron. J. Differential Equations, Vol. 2001 (2001), No. 59, 1-8.

[16] H. Deham and A. Djoudi, Periodic solutions for nonlinear differential equation with functional delay, Georgian Mathematical Journal 15 (2008), No. 4, 635-642.

[17] H. Deham and A. Djoudi, Existence of periodic solutions for neutral nonlinear differential equations withvariable delay, Electronic Journal of Differential Equations, Vol. 2010 (2010), No. 127, pp. 1-8.

[18] Y. M. Dib, M. R. Maroun and Y. N. Rafoul, Periodicity and stability in neutral nonlinear differential equations with functional delay, Electronic Journal of Differential Equations, Vol. 2005 (2005), No. 142, pp. 1-11.

[19] L. Ding, Z. Li, Periodicity and stability in neutral equations by krasnoselskii's fixed point theorem, Nonlinear Analysis: Real World Applications 11 (2010) 1220-1228.

[20] M. Fan and K. Wang, P. J. Y. Wong and R. P. Agarwal, Periodicity and stability in periodic $n$-species Lotka-Volterra competition system with feedback controls and deviating arguments, Acta Math. Sin. Engl. Ser. 19 (2003), no. 4, 801-822.

[21] H. I. Freedman, J. Wu, Periodic solutions of single-species models with periodic delay, SIAM J. Math. Anal. 23 (1992) 689-701.

[22] Y. Kuang, Delay Differential Equations with Application in Population Dynamics, Academic Press, New York, 1993.

[23] W. G. Li and Z. H. Shen, An constructive proof of the existence Theorem for periodic solutions of Duffng equations, Chinese Sci. Bull. 42 (1997), 1591-1595.

[24] Y. Liu, W. Ge, Positive periodic solutions of nonlinear Duffing equations with delay and variable coefficients, Tamsui Oxf. J. Math. Sci. 20 (2004) 235-255.

[25] F. Nouioua, A. Ardjouni, A. Djoudi, Periodic solutions for a third-order delay differential equation, Applied Mathematics E-Notes, 16 (2016), 210-221. 
[26] J. Ren, S. Siegmund and Y. Chen, Positive periodic solutions for third-order nonlinear differential equations, Electron. J. Differential Equations, Vol. 2011 (2011), No. 66, 1-19.

[27] D. R. Smart, Fixed Points Theorems, Cambridge University Press, Cambridge, 1980.

[28] Q. Wang, Positive periodic solutions of neutral delay equations (in Chinese), Acta Math. Sinica (N.S.) 6(1996), 789-795.

[29] Y. Wang, H. Lian and W. Ge, Periodic solutions for a second order nonlinear functional differential equation, Applied Mathematics Letters 20 (2007) 110-115.

[30] W. Zeng, Almost periodic solutions for nonlinear Duffing equations, Acta Math. Sinica (N.S.) 13(1997), 373-380.

[31] G. Zhang, S. Cheng, Positive periodic solutions of non autonomous functional differential equations depending on a parameter, Abstr. Appl. Anal. 7 (2002) 279-286. 